\title{
Article \\ When More Means Less: The Prognosis of Recurrent Acute Myocardial Infarctions
}

\author{
Ygal Plakht ${ }^{1,2, *(\mathbb{D})}$, Harel Gilutz ${ }^{3}\left(\mathbb{C}\right.$ and Arthur Shiyovich ${ }^{4,5}$ \\ 1 Department of Nursing, Faculty of Health Sciences, Ben-Gurion University of the Negev, \\ Beer-Sheva 8410501, Israel \\ 2 Department of Emergency Medicine, Soroka University Medical Center, Beer-Sheva 8489501, Israel \\ 3 Faculty of Health Sciences, Goldman Medical School, Ben-Gurion University of the Negev, \\ Beer-Sheva 8410501, Israel; Gilutz@bgu.ac.il \\ 4 Department of Cardiology, Rabin Medical Center, Petah Tikva 4941492, Israel; arthur.shiyovich@gmail.com \\ 5 Sackler Faculty of Medicine, Tel Aviv University, Tel Aviv 6139001, Israel \\ * Correspondence: Plakht@bgu.ac.il
}

check for updates

Citation: Plakht, Y.; Gilutz, H.; Shiyovich, A. When More Means Less: The Prognosis of Recurrent Acute Myocardial Infarctions. J. Clin. Med. 2021, 10, 5889. https://doi.org/ $10.3390 /$ jcm10245889

Academic Editors: Patrick De Boever and Francesco Onorati

Received: 16 November 2021 Accepted: 13 December 2021 Published: 15 December 2021

Publisher's Note: MDPI stays neutral with regard to jurisdictional claims in published maps and institutional affiliations.

Copyright: (c) 2021 by the authors. Licensee MDPI, Basel, Switzerland. This article is an open access article distributed under the terms and conditions of the Creative Commons Attribution (CC BY) license (https:/ / creativecommons.org/licenses/by/ $4.0 /)$.

\begin{abstract}
Recurrent acute myocardial infarctions (AMI) are common and associated with dismal outcomes. We evaluated the clinical characteristics and the prognosis of AMI survivors according to the number of recurrent AMIs (ReAMI) and the time interval of events (TI). A retrospective analysis of patients who survived following hospitalization with an AMI throughout 2002-2017 was conducted. The number of ReAMIs for each patient during the study period was recorded and classified based on following: 0 (no ReAMIs), 1, 2, $\geq 3$. Primary outcome: all-cause mortality up to 10 years post-discharge from the last AMI. A total of 12,297 patients (15,697 AMI admissions) were analyzed (age: $66.1 \pm 14.1$ years, $68 \%$ males). The mean number of AMIs per patient was $1.28 \pm 0.7$; the rates of $0,1,2, \geq 3$ ReAMIs were $81 \%, 13.4 \%, 3.6 \%$ and $1.9 \%$, respectively. The risk of mortality increased in patients with greater number of AMIs, HR $=1.666$ (95\% CI: 1.603-1.720, $p<0.001)$ for each additional event (study group), attenuated following adjustment for potential confounders, AdjHR $=1.135$ (95\% CI: 1.091-1.181, $p<0.001)$. Increased risk of mortality was found with short TI (<6-months), AdjHR = 2.205 (95\% CI: 1.418-3.429, $p<0.001$ ). The risk of mortality following AMI increased as the number of ReAMIs increased, and the TI between the events shortened. These findings should guide improved surveillance and management of this high-risk group of patients (i.e., ReAMI).
\end{abstract}

Keywords: recurrent acute myocardial infarction; mortality; prognosis; follow-up study

\section{Introduction}

Throughout the recent decades, multiple significant advancements in the management of patients with an acute myocardial infarction (AMI) were introduced and led to improved short- and long-term outcomes [1-4]. However, AMI survivors remain at increased risk of various adverse outcomes, including a recurrent AMI (ReAMI) [5,6]. Approximately $10 \%$ of patients are at risk of developing a ReAMI, accounting for about 200,000 cases per year in the United States [5-10]. Furthermore, ReAMIs are reported to be associated with worse outcomes $[5,6,9,10]$. However, previous reports often consisted of relatively short follow-up for ReAMIs or outcomes, were not contemporary and did not evaluate patients with multiple ReAMIs. The objective of the current study was to evaluate the clinical characteristics and the prognosis of AMI survivors with ReAMIs according to the number of events and the time interval between events (TI). 


\section{Materials and Methods}

\subsection{Study Population}

The present study includes a retrospective analysis of patients who survived following hospitalization with an AMI from Soroka University Medical Center (SUMC) throughout 2002-2017. SUMC is a tertiary referral center ( 1200 beds), singly serving the metropolitan area of Beer-Sheva, southern Israel (more than 500,000 residents). The last hospital admission during the period of data collection was defined as the index event. Patients were excluded due to the following criteria: (1) patients who were not residents of Southern District of Israel, (2) patients with a history of an MI prior to the earliest AMI event throughout the study period, (3) patients who died during their first admission during the study period and (4) AMI events that occurred within 28 days or less after a previous AMI. Such consecutive events might not represent a new separate event, but rather episodes of care related to the first event $[11,12]$. The ethics committee of SUMC approved the study, which was performed in accordance with the Helsinki Declaration.

\subsection{Data Sources and Classifications}

Data were obtained from the electronic medical files of SUMC (baseline characteristics) and the Ministry of the Interior Population Registry (mortality data). Baseline data of the index event included patients' demographic and clinical characteristics, laboratory, echocardiographic and angiographic data and management, as previously reported for the Soroka Acute Myocardial Infarction (SAMI) project [4]. Most baseline variables relating to past medical history were acquired using the International Classification of Diseases, Ninth Revision, Clinical Modification (ICD-9-CM) discharge codes. Specifically, AMI diagnosis was identified based on the ICD-9-CM codes: ST elevation MI (STEMI): 410.0 * -410.6 * and non-ST elevation MI (NSTEMI): $410.7^{*}-410.9^{*}$. In addition to the diagnostic codes, the diagnoses of anemia were grouped together with low hemoglobin blood levels. Patients were defined as having significant renal failure if they were either on hemodialysis/peritoneal dialysis or had an estimated glomerular filtration rate (eGFR) of $<30 \mathrm{~mL} / \mathrm{min} / 1.73 \mathrm{~m}^{2}$. The diagnosis of diabetes mellitus comprised of hemoglobin A1C levels $\geq 6.5 \%$, and dyslipidemia was considered if low-density lipoprotein level was $\geq 100 \mathrm{mg} / \mathrm{dL}$. Severe left ventricular (LV) dysfunction was defined as a left ventricular ejection fraction (LVEF) of $<30 \%$.

\subsection{Study Groups}

Patients were classified based on the number of AMI events throughout the study period as following: 0 (no ReAMI), 1, 2 and $\geq 3$. Additional analyses of the outcome ReAMIs, according to various TI of events, categorized as $<0.5$ year, $1,2,3,4$ and $\geq 5$ years, were performed.

\subsection{Follow-Up and Outcomes}

The primary outcome was all-cause mortality. The follow-up period to the primary outcome extended up to 10 years following the index event, with the last update being on 31 May 2021.

\subsection{Statistical Analysis}

Statistical analysis was executed using IBM SPSS Statistics 26 software. Patient characteristics were displayed as mean and standard deviation (SD) for continuous variables and $n$ and percent for the categorical data. Baseline characteristics were compared between the study groups using Chi-square test for linear trend and analysis of variance (ANOVA) for linear trend. Outcomes were compared between the study groups using the survival approach. The univariate analysis compared the risk of mortality with creation of survival functions (Kaplan-Meier) and using the log-rank test. In addition, regression analysis (Cox regression) was performed in order to estimate the relative risk for long-term mortality for the study groups. In this analysis, the group of patients with no ReAMI served as the refer- 
ence group. The multivariate analysis included Cox regression models. The parameters were introduced to the multivariate models using the stepwise method. The results of the models were presented as hazard ratios (HR)/adjusted hazard ratios (AdjHR) with 95\% confidence intervals (CI). For each test, $p<0.05$ was considered as statistically significant.

\section{Results}

\subsection{Study Population and Groups}

Throughout the study period, a total of 17,610 patients with at least one AMI were identified. Patients were excluded due to the following reasons: 2606 were not residents of the Southern District of Israel, 1627 had a prior AMI before the first AMI recorded during the study period, and 1080 died during the first admission. Thus, the final cohort for this study included 12,297 patients. The study flowchart is presented in the Supplementary Materials, Figure S1.

During the period of data collection, there were a total 15,697 admissions with an AMI. The mean number of AMIs per patient was $1.28 \pm 0.7$. The mean number of AMIs per patient among those who had a ReAMI was $1.46 \pm 0.91$. Overall, $9973(81 \%)$ patients did not have a ReAMI, 1647 (13.4\%) had one ReAMI, 446 (3.6\%) had two and 231 (1.9\%) had three or more ReAMIs. The distribution of the number of AMIs is presented in Table S1 of the Supplementary Materials.

The baseline characteristics of the study cohort (documented at the index hospitalization) according to the study groups are presented in Table 1. Patients in the groups with greater number of AMI events were older, with a higher proportion of women and minorities, and were more likely to present as NSTEMI compared with patients in the groups with lower number of AMI events. Furthermore, they had higher prevalence of most cardiovascular risk factors, excluding obesity and family history of ischemic heart disease, which were more prevalent among patients with lower number of AMI events. Moreover, patients with more ReAMI events had a more severe coronary artery disease, greater rate of congestive heart failure and severe left ventricular dysfunction. Additionally, they had higher prevalence of some non-cardiovascular comorbidities (i.e., chronic obstructive pulmonary disease (COPD), neurological disorders and anemia).

Table 1. The baseline characteristics of the study population (regarding to the index admission), by the number of recurrent acute myocardial infarction (ReAMI) events.

\begin{tabular}{|c|c|c|c|c|c|c|}
\hline \multirow{2}{*}{ Characteristic } & \multicolumn{4}{|c|}{ Number of ReAMIs } & \multirow{2}{*}{ Total } & \multirow{2}{*}{$p$-for-Trend } \\
\hline & 0 & 1 & 2 & $\geq 3$ & & \\
\hline$n$ & 9973 & 1647 & 446 & 231 & 12,297 & \\
\hline \multicolumn{7}{|l|}{ Demographics } \\
\hline Age, Mean (SD) & $65.19(14.10)$ & $69.18(13.82)$ & $70.96(13.32)$ & 73.01 (11.99) & $66.08(14.13)$ & $<0.001$ \\
\hline$<65$ & $5091(51.0)$ & $660(40.1)$ & $148(33.2)$ & $64(27.7)$ & $5963(48.5)$ & \\
\hline $65-75$ & $2181(21.9)$ & 377 (22.9) & $118(26.5)$ & $53(22.9)$ & $2729(22.2)$ & $<0.001$ \\
\hline$\geq 75$ & $2701(27.1)$ & $610(37.0)$ & $180(40.4)$ & $114(49.4)$ & $3605(29.3)$ & \\
\hline Sex, Males & $6833(68.5)$ & $1101(66.8)$ & $285(63.9)$ & $136(58.9)$ & 8355 (67.9) & $<0.001$ \\
\hline Ethnicity, Minorities & $1884(18.9)$ & $348(21.1)$ & $104(23.3)$ & $54(23.4)$ & $2390(19.4)$ & 0.001 \\
\hline \multicolumn{7}{|l|}{ Cardiac diseases } \\
\hline Cardiomegaly & $762(7.6)$ & $271(16.5)$ & $123(27.6)$ & $86(37.2)$ & $1242(10.1)$ & $<0.001$ \\
\hline Supraventricular arrhythmias & $1548(15.5)$ & $366(22.2)$ & $102(22.9)$ & $53(22.9)$ & $2069(16.8)$ & $<0.001$ \\
\hline $\mathrm{CHF}$ & $1491(15.0)$ & $457(27.7)$ & $192(43.0)$ & $129(55.8)$ & $2269(18.5)$ & $<0.001$ \\
\hline Pulmonary heart disease & $784(7.9)$ & $270(16.4)$ & $117(26.2)$ & $85(36.8)$ & $1256(10.2)$ & $<0.001$ \\
\hline AV block & $358(3.6)$ & $61(3.7)$ & $23(5.2)$ & $8(3.5)$ & $450(3.7)$ & 0.321 \\
\hline \multicolumn{7}{|l|}{ Cardiovascular risk factors } \\
\hline Renal diseases & $792(7.9)$ & $263(16.0)$ & $95(21.3)$ & $65(28.1)$ & $1215(9.9)$ & $<0.001$ \\
\hline Diabetes mellitus & $3796(38.1)$ & $872(52.9)$ & $270(60.5)$ & $166(71.9)$ & $5104(41.5)$ & $<0.001$ \\
\hline Dyslipidemia & $8011(80.3)$ & $1418(86.1)$ & $395(88.6)$ & $211(91.3)$ & $10,035(81.6)$ & $<0.001$ \\
\hline Hypertension & $5103(51.2)$ & $1060(64.4)$ & $339(76.0)$ & $200(86.6)$ & $6702(54.5)$ & $<0.001$ \\
\hline
\end{tabular}


Table 1. Cont.

\begin{tabular}{|c|c|c|c|c|c|c|}
\hline \multirow{2}{*}{ Characteristic } & \multicolumn{4}{|c|}{ Number of ReAMIs } & \multirow{2}{*}{ Total } & \multirow{2}{*}{$p$-for-Trend } \\
\hline & 0 & 1 & 2 & $\geq 3$ & & \\
\hline \multicolumn{7}{|l|}{ Cardiovascular risk factors } \\
\hline Obesity & $2263(22.7)$ & $353(21.4)$ & $77(17.3)$ & 43 (18.6) & $2736(22.2)$ & 0.003 \\
\hline Smoking & $4250(42.6)$ & $736(44.7)$ & $207(46.4)$ & $106(45.9)$ & $5299(43.1)$ & 0.026 \\
\hline PVD & $960(9.6)$ & $259(15.7)$ & $89(20.0)$ & $60(26.0)$ & $1368(11.1)$ & $<0.001$ \\
\hline Family history of IHD & $1008(10.1)$ & $172(10.4)$ & $33(7.4)$ & $13(5.6)$ & $1226(10.0)$ & 0.027 \\
\hline \multicolumn{7}{|l|}{ Other disorders } \\
\hline COPD & $746(7.5)$ & $229(13.9)$ & 87 (19.5) & $64(27.7)$ & $1126(9.2)$ & $<0.001$ \\
\hline Neurological disorders & 1559 (15.6) & $370(22.5)$ & $120(26.9)$ & $77(33.3)$ & $2126(17.3)$ & $<0.001$ \\
\hline Malignancy & $393(3.9)$ & $67(4.1)$ & $16(3.6)$ & $10(4.3)$ & $486(4.0)$ & 0.924 \\
\hline Anemia & $4080(40.9)$ & $815(49.5)$ & $250(56.1)$ & $134(58.0)$ & $5279(42.9)$ & $<0.001$ \\
\hline GI bleeding & $198(2.0)$ & $46(2.8)$ & $13(2.9)$ & $5(2.2)$ & $262(2.1)$ & 0.066 \\
\hline Schizophrenia/Psychosis & $182(1.8)$ & $27(1.6)$ & $8(1.8)$ & $7(3.0)$ & $224(1.8)$ & 0.574 \\
\hline Alcohol/drug addiction & $212(2.1)$ & $33(2.0)$ & $8(1.8)$ & $3(1.3)$ & $256(2.1)$ & 0.341 \\
\hline History of malignancy & $538(5.4)$ & $113(6.9)$ & $36(8.1)$ & $27(11.7)$ & $714(5.8)$ & $<0.001$ \\
\hline \multicolumn{7}{|l|}{ Clinical characteristics of AMI } \\
\hline Type of AMI, STEMI & $4691(47.0)$ & $447(27.1)$ & $78(17.5)$ & $18(7.8)$ & $5234(42.6)$ & $<0.001$ \\
\hline \multicolumn{7}{|l|}{ Results of echocardiography } \\
\hline Echocardiography performance & $8004(80.3)$ & $950(57.7)$ & $236(52.9)$ & $106(45.9)$ & $9296(75.6)$ & $<0.001$ \\
\hline Severe LV dysfunction & $756(9.4)$ & $150(15.8)$ & $64(27.1)$ & $33(31.1)$ & $1003(10.8)$ & $<0.001$ \\
\hline LV hypertrophy & $451(5.6)$ & $56(5.9)$ & $23(9.7)$ & $11(10.4)$ & $541(5.8)$ & 0.004 \\
\hline Mitral regurgitation & $389(4.9)$ & $76(8.0)$ & $23(9.7)$ & $11(10.4)$ & $499(5.4)$ & $<0.001$ \\
\hline Tricuspid regurgitation & $282(3.5)$ & $47(4.9)$ & $21(8.9)$ & $10(9.4)$ & $360(3.9)$ & $<0.001$ \\
\hline Pulmonary hypertension & $552(6.9)$ & $103(10.8)$ & $41(17.4)$ & $26(24.5)$ & $722(7.8)$ & $<0.001$ \\
\hline \multicolumn{7}{|l|}{ Results of angiography } \\
\hline Angiography performance & $7096(71.2)$ & $885(53.7)$ & $215(48.2)$ & $93(40.3)$ & $8289(67.4)$ & $<0.001$ \\
\hline $\begin{array}{c}\text { Measure of CAD, No or } \\
\text { non-significant }\end{array}$ & $390(5.5)$ & $37(4.2)$ & $8(3.7)$ & $6(6.5)$ & $441(5.3)$ & \multirow{4}{*}{$<0.001$} \\
\hline One vessel & $2122(29.9)$ & $185(20.9)$ & $32(14.9)$ & $12(12.9)$ & $2351(28.4)$ & \\
\hline Two vessels & $2008(28.3)$ & $258(29.2)$ & $58(27.0)$ & $22(23.7)$ & $2346(28.3)$ & \\
\hline Three vessels/ LM & $2576(36.3)$ & $405(45.8)$ & $117(54.4)$ & $53(57.0)$ & $3151(38.0)$ & \\
\hline \multicolumn{7}{|l|}{ Type of treatment } \\
\hline Noninvasive & $2523(25.3)$ & $658(40.0)$ & $208(46.6)$ & $132(57.1)$ & $3521(28.6)$ & \multirow{3}{*}{$<0.001$} \\
\hline PCI & $6182(62.0)$ & $851(51.7)$ & $213(47.8)$ & $92(39.8)$ & $7338(59.7)$ & \\
\hline CABG & $1268(12.7)$ & $138(8.4)$ & $25(5.6)$ & $7(3.0)$ & $1438(11.7)$ & \\
\hline
\end{tabular}

The data are presented as $n$ (\%) unless otherwise stated. AMI—acute myocardial infarction, AV—Atrioventricular (block), CABG—coronary artery bypass graft, $\mathrm{CAD}$ - coronary arteries disease, $\mathrm{CHF}$ - congestive heart failure, COPD—chronic obstructive pulmonary disease, GI-gastro-intestinal, IHD—ischemic heart disease, LM-left main (coronary artery), LV—left ventricular, PCI—percutaneous coronary intervention, PVD—-peripheral vascular disease, ReAMI—recurrent acute myocardial infarction, SD—standard deviation, STEMI—STelevation myocardial infarction.

\subsection{Follow-Up and Outcomes}

The median post-discharge follow-up was 2200 days ( $\sim 6$ years). During this period, $5278(42.9 \%)$ patients died (cumulative mortality 0.480 ). Mortality rates (cumulative mortality) were significantly higher as the number of admissions with AMI increased: $38.6 \%$ (cumulative mortality of 0.433$), 56.3 \%(0.636), 70.4 \%(0.804)$ and $82.7 \%(0.907)$ for the groups of $0,1,2$ and $\geq 3$ ReAMIs, respectively $(p$-for-trend $<0.001)$. Figure 1 presents the Kaplan-Meier survival functions for each study group. 


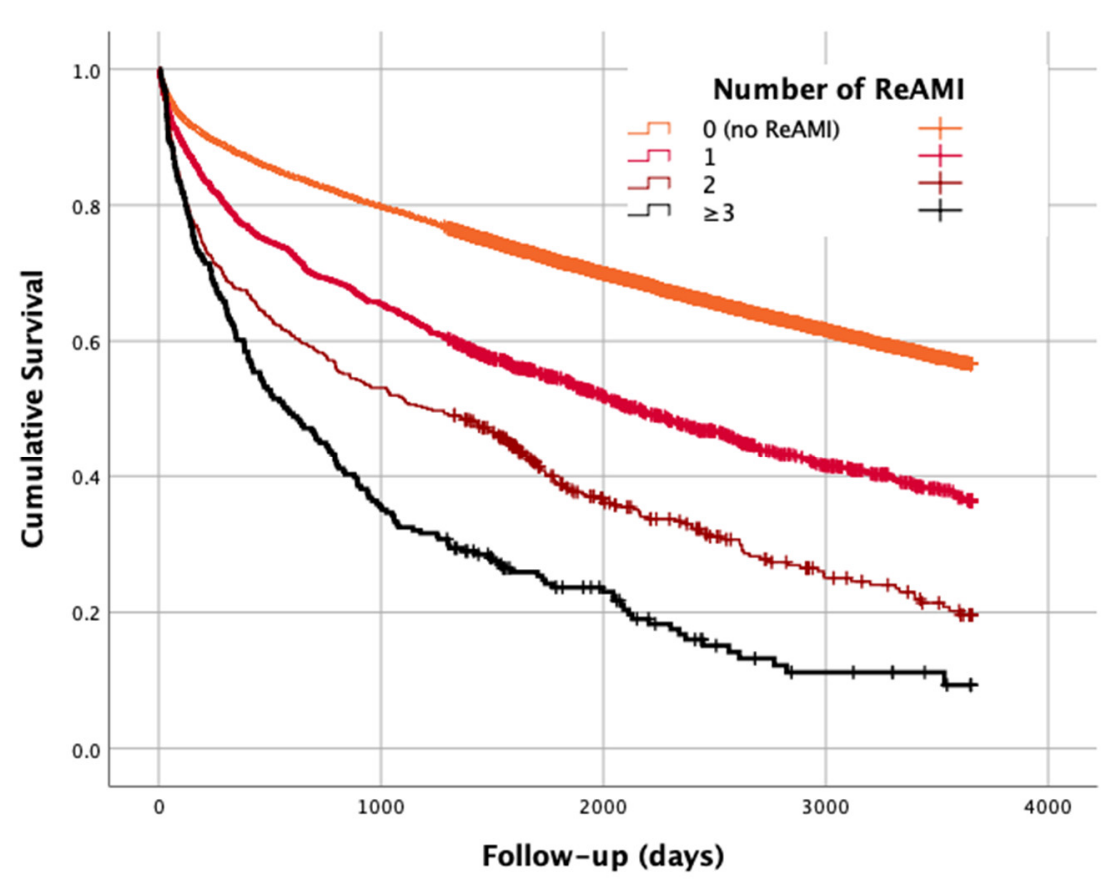

Figure 1. Survival function-cumulative mortality by the category of number of recurrent acute myocardial infarction (ReAMI) events. Log rank test, $p<0.001$.

The relative risks (HRs) for mortality for the groups of 1, 2 and $\geq 3$ ReAMI events were: 1.815 (95\% CI: 1.689-1.950), 2.783 (95\% CI: 2.480-3.123) and 4.143 (95\% CI: 3.579-4.794), respectively, as compared with the reference group ( $p<0.001$ for each) (Figure 2, blue line). With an increase in one group in the number of AMIs, the unadjusted HR for mortality was 1.666 (95\% CI: $1.603-1.720$, p-for-trend < 0.001).

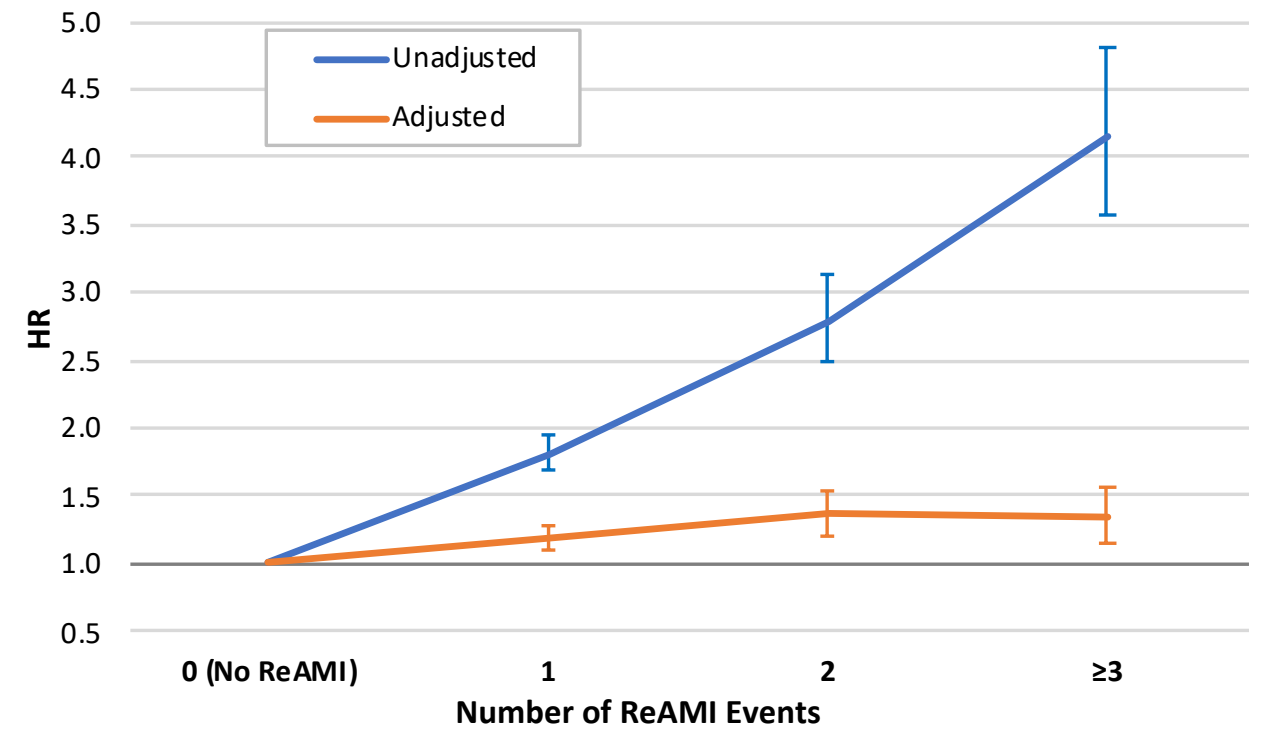

Figure 2. Relative risk for long-term mortality by the number of recurrent acute myocardial infarction (ReAMI) events. Blue line-unadjusted; red line-adjusted for the baseline characteristics (see Table 2 for the multivariate model). ReAMI-recurrent acute myocardial infarction, HR-hazard ratio. 
Table 2. Relationship between the number of recurrent acute myocardial infarction (ReAMI) events and the risk of long-term mortality, adjusted for the potential confounders. Multivariable model.

\begin{tabular}{|c|c|c|c|c|}
\hline Parameter & B (SE) & HR & $(95 \% \mathrm{CI})$ & $p$ \\
\hline \multicolumn{5}{|l|}{ Number of AMI Events: } \\
\hline 0 (no ReAMI) & & 1 (ref.) & & \\
\hline 2 & $0.167(0.039)$ & 1.181 & $(1.095 ; 1.274)$ & $<0.001$ \\
\hline 3 & $0.309(0.062)$ & 1.362 & $(1.207 ; 1.538)$ & $<0.001$ \\
\hline$\geq 3$ & $0.290(0.079)$ & 1.336 & $(1.144 ; 1.561)$ & $<0.001$ \\
\hline \multicolumn{5}{|l|}{ Age, Years: } \\
\hline$>65$ & & 1 (ref.) & & \\
\hline $65-75$ & $0.803(0.044)$ & 2.232 & $(2.048 ; 2.434)$ & $<0.001$ \\
\hline$\geq 75$ & $1.301(0.043)$ & 3.672 & $(3.373 ; 3.998)$ & $<0.001$ \\
\hline Sex, Male vs. Female & $-0.067(0.031)$ & 0.935 & $(0.881 ; 0.992)$ & 0.027 \\
\hline Cardiomegaly & $0.180(0.041)$ & 1.197 & $(1.105 ; 1.297)$ & $<0.001$ \\
\hline Supraventricular arrhythmias & $0.203(0.032)$ & 1.226 & $(1.150 ; 1.306)$ & $<0.001$ \\
\hline $\mathrm{CHF}$ & $0.241(0.033)$ & 1.273 & $(1.194 ; 1.357)$ & $<0.001$ \\
\hline Pulmonary heart disease & $0.094(0.042)$ & 1.098 & $(1.011 ; 1.193)$ & 0.027 \\
\hline Renal diseases & $0.388(0.038)$ & 1.474 & $(1.367 ; 1.588)$ & $<0.001$ \\
\hline Diabetes mellitus & $0.290(0.03)$ & 1.337 & $(1.261 ; 1.417)$ & $<0.001$ \\
\hline Dyslipidemia & $-0.194(0.034)$ & 0.823 & $(0.771 ; 0.880)$ & $<0.001$ \\
\hline Hypertension & $-0.076(0.031)$ & 0.927 & $(0.873 ; 0.984)$ & 0.013 \\
\hline Obesity & $-0.120(0.037)$ & 0.887 & $(0.824 ; 0.954)$ & 0.001 \\
\hline PVD & $0.333(0.037)$ & 1.395 & $(1.297 ; 1.501)$ & $<0.001$ \\
\hline Family history of IHD & $-0.382(0.093)$ & 0.682 & $(0.569 ; 0.818)$ & $<0.001$ \\
\hline COPD & $0.525(0.040)$ & 1.691 & $(1.565 ; 1.827)$ & $<0.001$ \\
\hline Neurological disorders & $0.448(0.032)$ & 1.564 & $(1.470 ; 1.665)$ & $<0.001$ \\
\hline Malignancy & $0.591(0.055)$ & 1.806 & $(1.621 ; 2.011)$ & $<0.001$ \\
\hline Anemia & $0.295(0.031)$ & 1.343 & $(1.264 ; 1.427)$ & $<0.001$ \\
\hline GI bleeding & $0.228(0.077)$ & 1.256 & $(1.080 ; 1.460)$ & 0.003 \\
\hline Schizophrenia/Psychosis & $0.435(0.082)$ & 1.546 & $(1.316 ; 1.816)$ & $<0.001$ \\
\hline Alcohol/drug addiction & $0.550(0.095)$ & 1.734 & $(1.439 ; 2.088)$ & $<0.001$ \\
\hline Type of AMI: STEMI vs. NSTEMI & $-0.148(0.034)$ & 0.863 & $(0.807 ; 0.922)$ & $<0.001$ \\
\hline LOS: $>7$ days vs. $\leq 7$ days & $0.161(0.031)$ & 1.174 & $(1.105 ; 1.247)$ & $<0.001$ \\
\hline \multicolumn{5}{|l|}{ Type of treatment: } \\
\hline Noninvasive & & 1 (ref.) & & \\
\hline PCI & $-0.661(0.035)$ & 0.517 & $(0.482 ; 0.553)$ & $<0.001$ \\
\hline CABG & $-1.055(0.062)$ & 0.348 & $(0.308 ; 0.393)$ & $<0.001$ \\
\hline Severe LV dysfunction & $0.422(0.048)$ & 1.526 & $(1.389 ; 1.676)$ & $<0.001$ \\
\hline LV hypertrophy & $0.189(0.064)$ & 1.208 & $(1.067 ; 1.368)$ & 0.003 \\
\hline Mitral regurgitation & $0.223(0.060)$ & 1.250 & $(1.112 ; 1.405)$ & $<0.001$ \\
\hline Pulmonary hypertension & $0.219(0.055)$ & 1.244 & $(1.118 ; 1.385)$ & $<0.001$ \\
\hline Year of the index event (one year increase) & $-0.013(0.004)$ & 0.987 & $(0.980 ; 0.994)$ & $<0.001$ \\
\hline
\end{tabular}

AMI-acute myocardial infarction, B-regression coefficient, CABG-coronary artery bypass graft, CHFcongestive heart failure, $\mathrm{CI}$ - confidence interval, $\mathrm{COPD}$ — chronic obstructive pulmonary disease, $\mathrm{HR}$ - hazard ratio, GI-gastro-intestinal, IHD—ischemic heart disease, LV—left ventricular, NSTEMI—non-ST-elevation myocardial infarction, PCI - percutaneous coronary intervention, PVD—peripheral vascular disease, ReAMIrecurrent acute myocardial infarction, ref.-reference (group), SE—standard deviation, STEMI—ST-elevation myocardial infarction.

\subsection{Multivariable Analysis}

Following multivariate adjustment to potential confounders (Table 2 and Figure 2, red line), AdjHRs for mortality for the groups were: 1.181, 1.362 and 1.336 for 1,2 and $\geq 3$ ReAMI events, respectively, as compared with the reference group. The AdjHR for mortality with an increase in one group in the number of AMIs was 1.135 (95\% CI: 1.091-1.181, $p$-fortrend $<0.001$ ).

\subsection{Sub-Group Analysis}

In order to evaluate the risk of mortality according to the various TIs, the groups of 2 ReAMIs and $\geq 3$ ReAMIs were merged into the category of $\geq 2$ ReAMIs (due to the small 
size of the latter group). The distribution of the TIs by the number of the AMI groups are presented in Supplementary Table S2.

The rates of the TIs between the AMIs in the different AMI number groups are presented in Supplementary Table S2. For every category of the number of AMIs, a significant trend ( $p$-for-trend $<0.001$ for each) of decrease in the risk of mortality was found when the TI increased. However, when two events occurred within half a year or less, the risk of mortality was greater compared with $0.5-1$-year TI, borderline statistical significance $(p=0.053)$.

The adjusted risks for mortality according to the number of AMI events and TIs are presented in Figure 3 and Supplementary Table S3. Following adjustment, the highest risk of long-term mortality was observed for $\geq 2$ ReAMIs that occurred within less than 0.5 year: AjdHR = 2.205 (95\% CI: 1.418-3.429) and 0.5-1-year: AjdHR = 1.933 (95\% CI: 1.448-2.580), $(p<0.001$ for each) and was significantly lower for longer TIs (as compared with the group with no ReAMI). However, no significant trend for relative risk of mortality related to TI in the group with 1 ReAMI event was found.

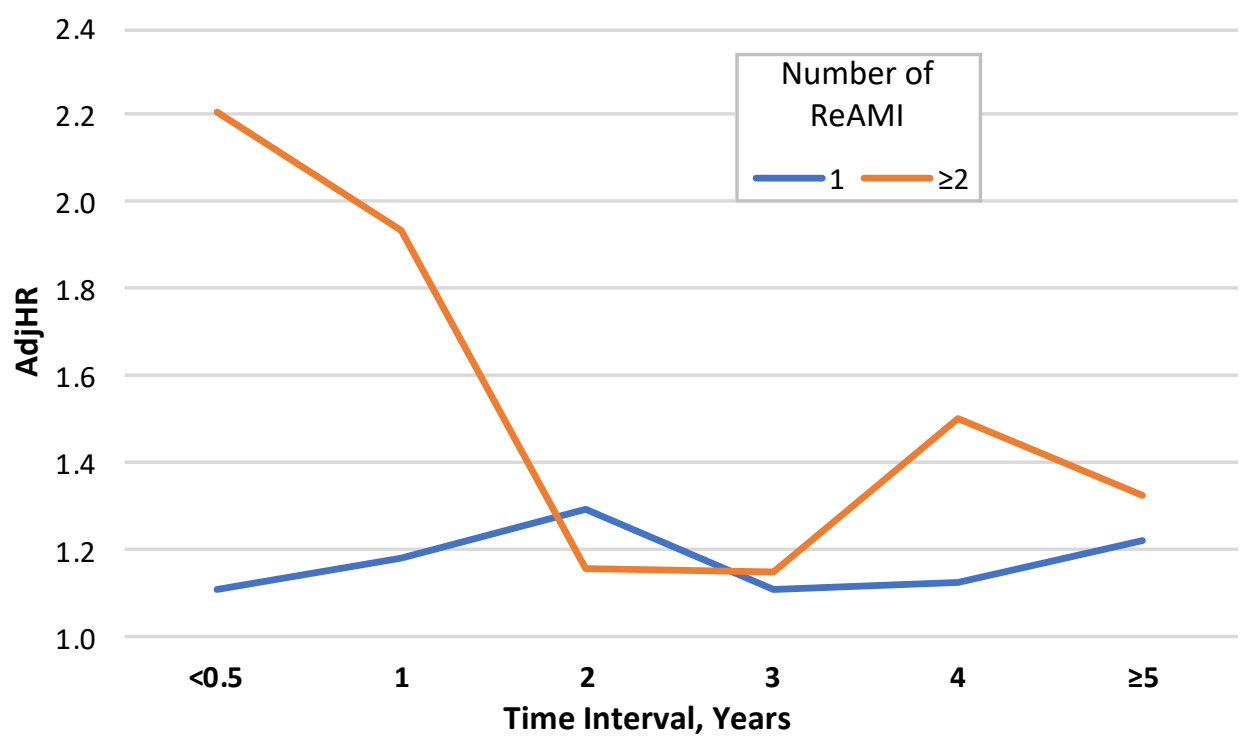

Figure 3. The adjusted relative risks for mortality according to the number of recurrent acute myocardial infarction (ReAMI) events * and the time intervals (see Table S3 for the full multivariable model). * Reference group-no recurrent acute myocardial infarction (ReAMI) events. AdjHRadjusted hazard ratio.

\section{Discussion}

The present study evaluated patient characteristics and outcomes in patients with ReAMIs according to the number of events and their TI. The main findings include: (1) the risk of mortality increased significantly with the increase in the number of AMIs, especially $\geq 3$ ReAMI events; (2) following adjustment to potential confounders, the risk of mortality with ReAMI attenuated, yet it remained significantly increased; and (3) considering the TI of events, the highest risk was when $\geq 2$ ReAMIs occurred during a period of less than 1 year.

The rate of patients with ReAMI in the current study ( $\geq 2$ admissions with an AMI) was $18.9 \%$ : higher compared with some previous studies $[5,6,11,13,14]$, nearly identical to a study by Viveiros et al. [15] and lower from that reported by Motivala et al. [9]. These differences stem mostly from the length of the follow-up period and the methodology used in the current study, which focused on analyzing patients according to the exact number of AMIs and not only on separating first events from ReAMIs. Additional reasons could be the real-life, highly unrepresentative cohort, with period of inclusion beginning almost two decades ago (higher rate of MACE, including ReAMIs, at that period) $[4,16]$. 
The finding that patients with ReAMI are older, with higher prevalence of most cardiovascular risk factors and more severe coronary disease, yet a decreased rate of revascularization, is also consistent with previous reports $[5,6,11,13,14]$. However, the current study also adds analysis with the number of ReAMIs that display a gradual ("dose response"-like) response to most variables.

The finding of this study that the risk of mortality increased significantly with the increase in the number of ReAMIs is in line with the findings of previous studies $[5,6,11,13,14,17]$. Although Motivala et al. [9] reported similar in-hospital mortality among patients with ReAMI vs. those with a first AMI, mortality six months following discharge was significantly higher among patients with a ReAMI. This study extends previous literature in the investigation of more than one ReAMI and demonstrates a gradual increase in risk with every event (considering also the TI between the events), the prolonged followup period, the unrepresentative population and the relatively high number of investigated confounders used for adjustment and indeed attenuating the independent risk significantly. The reasons for the increased risk of mortality with increased numbers of AMIs are largely unknown. However, several potential explanations can be suggested. First, patients with ReAMIs are older, with worse risk profile and higher prevalence of cardiovascular risk factors, and both cardiac and non-cardiac comorbidities, that increase their risk. Although we adjusted for age and most risk factors, some unaccounted-for comorbidities probably still contribute to their increased risk. Second, these patients were less intensively treated with a more conservative approach, which could also accelerate earlier recurrence [18]. The latter mechanism is also supported by previous literature showing worse outcome for patients presenting with apparently a first AMI but diagnosed with a previous silent AMI that was not treated [19]. Furthermore, with the increasing number of ReAMI, we saw a trend of decreasing number of coronary angiographies and percutaneous coronary interventions (PCI). Although the latter might be partially explained by a wider use of coronary artery bypass graft (CABG) with increasing ReAMIs, we believe this somewhat conservative management also stems from higher rate of NSTEMI, older and more frail patients, greater rate of chronic kidney disease and the fact that the study includes patients over a long period of time, starting from almost two decades ago, when a conservative approach was more prevalent. Third, ReAMI is often associated with reduced adherence with guidelines and compliance with optimal medical therapy, despite the strong evidence supporting the efficacy of such therapy, specifically in patients with ReAMI [5,20-22]. Moreover, although studies exploring the specific therapy of patients with ReAMI are insufficient, most recommendations support a stricter approach in follow-up and control of cardiovascular risk factors [23,24]. Fourth, the more severe coronary disease and particularly the increased prevalence of multivessel disease (as found in ReAMIs in the current study) have been reported as risk factors for ReAMI $[6,16]$.

The increased risk with every ReAMI (which were usually chronologically later in our cohorts than initial events) should also be considered in the context of significantly improved outcomes in patients with AMI throughout the last decades, following major upgrades in therapy [1-4]. Thus, this risk might actually be underestimated in the current study.

The finding of the current study that the risk of mortality increases with more AMIs within shorter TI is in line with previous studies showing worse short-term mortality in cases of ReAMI $[5,15,18,25]$. This could be explained by the higher rate of stent thrombosis, which is associated with dismal outcomes, particularly during the relatively early period $[6,15]$. Nevertheless, non-stent-related ReAMI is also more common during the early period [15]. Additional reasons explaining worse outcomes when ReAMI occur during the early period following AMI include increased rates of various potentially lethal complications (e.g., mechanical complications), increased vulnerability shortly following hospitalization, delayed prescription filling and increased excessive inflammation, either as a response to the AMI or for other reasons [26-30]. 


\section{Limitations}

Our findings need to be interpreted in the context of certain limitations. First, our study is retrospective and observational and shares the limitations of such design. Second, although our multivariate models included adjustment for multiple potential confounders, unaccounted-for confounders might still affect the observed differences between the groups. Third, we did not have information regarding the quality of care upon discharge and thereafter, in particular the rate of prescription and usage of guideline-recommended medications (e.g., stating, anti-platelet agents, angiotensin-converting enzyme (ACE) inhibitors, etc.). Fourth, the causes of death (specifically cardiac vs. non-cardiac) were not included in the current study and might have resulted in underestimation of the rates of ReAMI. Furthermore, it is possible that some of the mortalities, especially sudden death, were actually a ReAMI which was not counted as such in the current study. Fifth, silent AMIs were not systematically evaluated and recorded in the current study, which might have underestimated the rate of ReAMI. Sixth, as we used the ICD-9-CM diagnosis codes for most of the baseline characteristics, consequently, we relied on the treating physicians' partial bias (i.e., diagnosis or coding bias), which might have occurred in some cases. However, we felt that overruling and "second guessing" the treating physician in such cases might introduce more inaccuracies. Seventh, we excluded ReAMIs within 28 days or less from the previous events, as they might not have represented a separate event; however, this might have underestimated the incidence of ReAMI in our cohort.

\section{Conclusions}

The risk of mortality following AMI increases with the increase in the number of ReAMI events and shorter TI of AMIs, with the highest risk being when $\geq 3$ AMIs occur during a period of less than 1 year. These findings should guide more intense surveillance and management of this high-risk group of patients (i.e., ReAMI with short term), both upon hospital admission and post-discharge. Further investigation of unique strategies and treatment goals for patients with ReAMI are warranted to improve their outcomes.

Supplementary Materials: The following are available online at https: / www.mdpi.com/article/ 10.3390/jcm10245889/s1. Figure S1: The study flowchart. Table S1: Distribution of the number of recurrent acute myocardial infarction (ReAMI) events in the study population. Table S2: Distribution of the time intervals by the number of recurrent acute myocardial infarction (ReAMI) events. Table S3: The adjusted relative risks (adjusted hazard ratios-AdjHR) for mortality according to the number of recurrent acute myocardial infarction (ReAMI) events and the time intervals. Multivariable model.

Author Contributions: Y.P., H.G. and A.S. contributed to the conception and design of the work. Y.P. contributed to the acquisition and analysis of data for the work. A.S. and H.G. contributed to data interpretation. A.S. drafted the manuscript. Y.P. and H.G. critically revised the manuscript. All authors gave final approval and agreed to be accountable for all aspects of work, ensuring integrity and accuracy. All authors have read and agreed to the published version of the manuscript.

Funding: This research received no external funding.

Institutional Review Board Statement: The study was conducted according to the guidelines of the Declaration of Helsinki and approved by the Institutional Ethics Committee of Soroka University Medical Center (protocol code SOR-0319-16, extended 3 December 2021).

Informed Consent Statement: Patient consent was waived due to anonymous retrospective data collection.

Data Availability Statement: The data presented in this study are available on request from the corresponding author. The data are not publicly available due to privacy.

Conflicts of Interest: The authors declare no conflict of interest. 


\section{References}

1. Peterson, E.D.; Shah, B.R.; Parsons, L.; Pollack, C.V., Jr.; French, W.J.; Canto, J.G.; Gibson, C.M.; Rogers, W.J. Trends in quality of care for patients with acute myocardial infarction in the National Registry of Myocardial Infarction from 1990 to 2006 . Am. Heart J. 2008, 156, 1045-1055. [CrossRef]

2. Braunwald, E.; Antman, E.M.; Beasley, J.W.; Califf, R.M.; Cheitlin, M.D.; Hochman, J.S.; Jones, R.H.; Kereiakes, D.; Kupersmith, J.; Levin, T.N.; et al. ACC/AHA 2002 guideline update for the management of patients with unstable angina and non-ST-segment elevation myocardial infarction-Summary article: A report of the American College of Cardiology/American Heart Association task force on practice guidelines (Committee on the Management of Patients with Unstable Angina). J. Am. Coll. Cardiol. 2002, 40, 1366-1374. [CrossRef]

3. Kushner, F.G.; Hand, M.; Smith, S.C., Jr.; King, S.B., 3rd; Anderson, J.L.; Antman, E.M.; Bailey, S.R.; Bates, E.R.; Blankenship, J.C.; Casey, D.; et al. 2009 focused updates: ACC/AHA guidelines for the management of patients with ST-elevation myocardial infarction (updating the 2004 guideline and 2007 focused update) and ACC/AHA/SCAI guidelines on percutaneous coronary intervention (updating the 2005 guideline and 2007 focused update) a report of the American College of Cardiology Foundation/American Heart Association Task Force on Practice Guidelines. J. Am. Coll. Cardiol. 2009, 54, 2205-2241. [CrossRef] [PubMed]

4. Plakht, Y.; Gilutz, H.; Shiyovich, A. Temporal trends in acute myocardial infarction: What about survival of hospital survivors? Disparities between STEMI \& NSTEMI remain. Soroka acute myocardial infarction II (SAMI-II) project. Int. J. Cardiol. 2016, 203, 1073-1081. [CrossRef] [PubMed]

5. Song, J.; Murugiah, K.; Hu, S.; Gao, Y.; Li, X.; Krumholz, H.M.; Zheng, X. Incidence, predictors, and prognostic impact of recurrent acute myocardial infarction in China. Heart 2020, 107, 313-318. [CrossRef] [PubMed]

6. Stone, S.G.; Serrao, G.W.; Mehran, R.; Tomey, M.I.; Witzenbichler, B.; Guagliumi, G.; Peruga, J.Z.; Brodie, B.R.; Dudek, D.; Möckel, M.; et al. Incidence, predictors, and implications of reinfarction after primary percutaneous coronary intervention in ST-segment-elevation myocardial infarction: The Harmonizing Outcomes with Revascularization and Stents in Acute Myocardial Infarction Trial. Circ. Cardiovasc. Interv. 2014, 7, 543-551. [CrossRef] [PubMed]

7. Benjamin, E.J.; Muntner, P.; Alonso, A.; Bittencourt, M.S.; Callaway, C.W.; Carson, A.P.; Chamberlain, A.M.; Chang, A.R.; Cheng, S.; Das, S.R.; et al. Heart Disease and Stroke Statistics-2019 Update: A Report From the American Heart Association. Circulation 2019, 139, e56-e528. [CrossRef] [PubMed]

8. Jernberg, T.; Hasvold, P.; Henriksson, M.; Hjelm, H.; Thuresson, M.; Janzon, M. Cardiovascular risk in post-myocardial infarction patients: Nationwide real world data demonstrate the importance of a long-term perspective. Eur. Heart J. 2015, 36, 1163-1170. [CrossRef] [PubMed]

9. Motivala, A.A.; Tamhane, U.; Ramanath, V.S.; Saab, F.; Montgomery, D.G.; Fang, J.; Kline-Rogers, E.; May, N.; Ng, G.; Froehlich, J.; et al. A prior myocardial infarction: How does it affect management and outcomes in recurrent acute coronary syndromes? Clin. Cardiol. 2008, 31, 590-596. [CrossRef]

10. Wagner, S.; Burczyk, U.; Schiele, R.; Bergmeier, C.; Rustige, J.; Gottwik, M.; Senges, J. The 60 Minutes Myocardial Infarction Project. Characteristics on admission and clinical outcome in patients with reinfarction compared to patients with a first infarction. Eur. Heart J. 1998, 19, 879-884. [CrossRef]

11. Wang, Y.; Leifheit, E.; Normand, S.T.; Krumholz, H.M. Association Between Subsequent Hospitalizations and Recurrent Acute Myocardial Infarction within 1 Year after Acute Myocardial Infarction. J. Am. Heart Assoc. 2020, 9, e014907. [CrossRef]

12. Mendis, S.; Thygesen, K.; Kuulasmaa, K.; Giampaoli, S.; Mähönen, M.; Blackett, K.N.; Lisheng, L.; Writing group on behalf of the participating experts of the WHO consultation for revision of WHO definition of myocardial infarction. World Health Organization definition of myocardial infarction: 2008-09 revision. Int. J. Epidemiol. 2011, 40, 139-146. [CrossRef] [PubMed]

13. Nakatani, D.; Sakata, Y.; Suna, S.; Usami, M.; Matsumoto, S.; Shimizu, M.; Sumitsuji, S.; Kawano, S.; Ueda, Y.; Hamasaki, T.; et al. Incidence, predictors, and subsequent mortality risk of recurrent myocardial infarction in patients following discharge for acute myocardial infarction. Circ. J. 2013, 77, 439-446. [CrossRef] [PubMed]

14. Mal, K.; Awan, I.D.; Shaukat, F. Evaluation of Risk Factors Associated with Reinfarction: A Multicenter Observational Study. Cureus 2019, 11, e6063. [CrossRef] [PubMed]

15. Monteiro, A.V.; Ramos, R.; Fiarresga, A.; de Sousa, L.; Cacela, D.; Patrício, L.; Bernardes, L.; Soares, C.; Ferreira, R.C. Timing and long-term prognosis of recurrent MI after primary angioplasty: Stent thrombosis vs. non-stent-related reinfarction. Herz 2017, 42, 186-193. [CrossRef]

16. Shiyovich, A.; Shlomo, N.; Cohen, T.; Iakobishvili, Z.; Kornowski, R.; Eisen, A. Temporal trends of patients with acute coronary syndrome and multi-vessel coronary artery disease-from the ACSIS registry. Int. J. Cardiol. 2020, 304, 8-13. [CrossRef]

17. Cao, C.-F.; Li, S.-F.; Chen, H.; Song, J.-X. Predictors and in-hospital prognosis of recurrent acute myocardial infarction. J. Geriatr. Cardiol. 2016, 13, 836-839. [CrossRef]

18. Nair, R.; Johnson, M.; Kravitz, K.; Huded, C.; Rajeswaran, J.; Anabila, M.; Blackstone, E.; Menon, V.; Lincoff, A.M.; Kapadia, S.; et al. Characteristics and Outcomes of Early Recurrent Myocardial Infarction After Acute Myocardial Infarction. J. Am. Heart Assoc. 2021, 10, e019270. [CrossRef] [PubMed]

19. Amier, R.P.; Smulders, M.W.; van der Flier, W.M.; Bekkers, S.C.; Zweerink, A.; Allaart, C.P.; Demirkiran, A.; Roos, S.T.; Teunissen, P.; Appelman, Y.; et al. Long-Term Prognostic Implications of Previous Silent Myocardial Infarction in Patients Presenting with Acute Myocardial Infarction. JACC Cardiovasc. Imaging 2018, 11, 1773-1781. [CrossRef] 
20. Bansilal, S.; Castellano, J.M.; Garrido, E.; Wei, H.G.; Freeman, A.; Spettell, C.; Garcia-Alonso, F.; Lizano, I.; Arnold, R.J.; Rajda, J.; et al. Assessing the Impact of Medication Adherence on Long-Term Cardiovascular Outcomes. J. Am. Coll. Cardiol. 2016, 68, 789-801. [CrossRef]

21. Du, L.; Cheng, Z.; Zhang, Y.; Li, Y.; Mei, D. The impact of medication adherence on clinical outcomes of coronary artery disease: A meta-analysis. Eur. J. Prev. Cardiol. 2017, 24, 962-970. [CrossRef] [PubMed]

22. Zhang, D.; Song, X.; Raposeiras-Roubín, S.; Abu-Assi, E.; Henriques, J.P.S.; D’Ascenzo, F.; Saucedo, J.; González-Juanatey, J.R.; Wilton, S.B.; Kikkert, W.J.; et al. The impact of optimal medical therapy on patients with recurrent acute myocardial infarction: Subanalysis from the BleeMACS study. Int. J. Cardiol. 2020, 318, 1-6. [CrossRef]

23. Visseren, F.L.J.; Mach, F.; Smulders, Y.M.; Carballo, D.; Koskinas, K.C.; Bäck, M.; Benetos, A.; Biffi, A.; Boavida, J.-M.; Capodanno, D.; et al. 2021 ESC Guidelines on cardiovascular disease prevention in clinical practice. Eur. Heart J. 2021, 42, 3227-3337. [CrossRef] [PubMed]

24. Mach, F.; Baigent, C.; Catapano, A.L.; Koskinas, K.C.; Casula, M.; Badimon, L.; Chapman, M.J.; De Backer, G.G.; Delgado, V.; Ference, A.B.; et al. 2019 ESC/EAS Guidelines for the management of dyslipidaemias: Lipid modification to reduce cardiovascular risk. Eur. Heart J. 2020, 41, 111-188. [CrossRef] [PubMed]

25. Shiraishi, J.; Kohno, Y.; Sawada, T.; Takeda, M.; Arihara, M.; Hyogo, M.; Yagi, T.; Shima, T.; Okada, T.; Nakamura, T.; et al. Predictors of in-hospital outcome after primary percutaneous coronary intervention for recurrent myocardial infarction. Circ. J. 2008, 72, 1225-1229. [CrossRef]

26. Gong, F.F.; Vaitenas, I.; Malaisrie, S.C.; Maganti, K. Mechanical Complications of Acute Myocardial Infarction: A Review. JAMA Cardiol. 2021, 6, 341-349. [CrossRef]

27. Krumholz, H.M. Post-Hospital Syndrome-An Acquired, Transient Condition of Generalized Risk. N. Engl. J. Med. 2013, 368, 100-102. [CrossRef]

28. Ho, P.M.; Tsai, T.T.; Maddox, T.M.; Powers, J.D.; Carroll, N.M.; Jackevicius, C.; Go, A.S.; Margolis, K.L.; DeFor, T.A.; Rumsfeld, J.S.; et al. Delays in filling clopidogrel prescription after hospital discharge and adverse outcomes after drug-eluting stent implantation: Implications for transitions of care. Circ. Cardiovasc. Qual. Outcomes 2010, 3, 261-266. [CrossRef]

29. Ong, S.B.; Hernández-Reséndiz, S.; Crespo-Avilan, G.E.; Mukhametshina, R.T.; Kwek, X.Y.; Cabrera-Fuentes, H.A.; Hausenloy, D.J. Inflammation following acute myocardial infarction: Multiple players, dynamic roles, and novel therapeutic opportunities. Pharmacol. Ther. 2018, 186, 73-87. [CrossRef] [PubMed]

30. Frangogiannis, N.G. The inflammatory response in myocardial injury, repair, and remodelling. Nat. Rev. Cardiol. 2014, 11, 255-265. [CrossRef] 